\title{
Fusing Eye Movements and Observer Narratives for Expert-Driven Image-Region Annotations
}

\author{
Preethi Vaidyanathan* \\ Jeff B. Pelz ${ }^{\dagger}$ \\ Carlson Imaging Science, RIT
}

\author{
Emily Prud'hommeaux ${ }^{\ddagger}$ \\ Cecilia Ovesdotter Alm ${ }^{\S}$ \\ College of Liberal Arts, RIT
}

\author{
Anne R. Haake ${ }^{\text {I }}$ \\ College of Computing and \\ Information Sciences RIT
}

\begin{abstract}
Human image understanding is reflected by individuals' visual and linguistic behaviors, but the meaningful computational integration and interpretation of their multimodal representations remain a challenge. In this paper, we expand a framework for capturing image-region annotations in dermatology, a domain in which interpreting an image is influenced by experts' visual perception skills, conceptual domain knowledge, and task-oriented goals. Our work explores the hypothesis that eye movements can help us understand experts' perceptual processes and that spoken language descriptions can reveal conceptual elements of image inspection tasks. We cast the problem of meaningfully integrating visual and linguistic data as unsupervised bitext alignment. Using alignment, we create meaningful mappings between physicians' eye movements, which reveal key areas of images, and spoken descriptions of those images. The resulting alignments are then used to annotate image regions with medical concept labels. Our alignment accuracy exceeds baselines using both exact and delayed temporal correspondence. Additionally, comparison of alignment accuracy between a method that identifies clusters in the images based on eye movement vs. a method that identifies clusters using image features suggests that the two approaches perform well on different types of images and concept labels. This suggests that an image annotation framework should integrate information from more than one technique to handle heterogeneous images. We also investigate the performance of the proposed aligner for dermatological primary morphology concept labels, as well as for lesion size or type and distribution-based categories of images.
\end{abstract}

Keywords: image annotation, bitext alignment

Concepts: $\bullet$ Computing methodologies $\rightarrow$ Scene understanding; Visual content-based indexing and retrieval;

\section{Introduction}

Semantic image annotation plays a key role in developing sophisticated image-information systems but remains a difficult research challenge. The "semantic-gap" between low-level features and high-level semantic concepts limits the use of existing image

\footnotetext{
*pxv1621@rit.edu

†pelz@cis.rit.edu

¥emilypx@rit.edu

$\S$ coagla@rit.edu

ฯarhics@ rit.edu
}

Permission to make digital or hard copies of all or part of this work for personal or classroom use is granted without fee provided that copies are not made or distributed for profit or commercial advantage and that copies bear this notice and the full citation on the first page. Copyrights for components of this work owned by others than ACM must be honored. Abstracting with credit is permitted. To copy otherwise, or republish, to post on servers or to redistribute to lists, requires prior specific permission and/or a fee. Request permissions from permissions@acm.org. (c) 2016 ACM.

ETRA '16, March 14-17, 2016, Charleston, SC, USA

ISBN: 978-1-4503-4125-7/16/03

DOI: http://dx.doi.org/10.1145/2857491.2857542 processing algorithms to understand or retrieve images at a meaningful level [Smeulders et al. 2000; Liu et al. 2007; Zhang et al. 2012]. This work builds on methods for integrating experts' eye movements, narratives, and image features to achieve semantic annotations of image regions.

High-level domain knowledge and experience enable expert observers to understand and process images at a semantic level that is difficult for a computer [Shanteau 1992; Goldstone 1998]. Understanding experts' ability to identify image objects and regions is crucial in advancing various image-based application systems. When expert observers communicate their understanding and reasoning of images, spoken language is the most natural and convenient instrument. Vaidyanathan et al. [2015a; 2015b] use a grid-based and mean-shift clustering techniques in conjunction with bitext alignment to align eye movements and transcribed narratives to integrate experts' perceptual and conceptual knowledge. We build on this framework to include image features that will be useful in building image-based application systems.

Improving systems involving cognitively complex tasks such as medical image diagnosis requires understanding human information processing mechanisms [Hoffman and Fiore 2007]. Eye movements over an image reveal important regions and their relation to one another from the observer's perspective. Also, co-captured image descriptions provide relevant meaning, particularly special knowledge and experience that domain experts possess. Even when the visual and linguistic information are co-captured, they cannot be assumed to have one-to-one or fixed-delay temporal correspondence, thereby demanding sophisticated methods for integrating them [Meyer et al. 1998; van der Meulen 2003; Griffin 2004].

The bitext word alignment method, initially developed for machine translation, aligns the linguistic units (e.g., words) of a sentence in one language to their corresponding translations in another language. Analogously, in the multimodal problem, one can consider the eye movements on images as the visual language comprising visual units of analysis and the transcribed narratives as the linguistic units of analysis. Association of words and sentences with images, objects and image regions, and videos has been investigated extensively [Forsyth et al. 2009; Kuznetsova et al. 2013; Kong et al. 2014; Socher et al. 2014; Thomason et al. 2014]. We believe semantic annotation of image regions can be strengthened by combining perceptual and conceptual information via the integration of eye movements, narratives, and image feature content. Expanding on our previous work, we use bitext alignment to perform this integration. The resulting alignments are used to annotate important image regions with appropriate lexical concepts. The novelty lies in extending our work to involve image features that ultimately are the basis of any image-based application system. Our main contributions are as follows:

1. We show that expert elicited eye movements and descriptions jointly provide information that otherwise would be insufficient when considered separately.

2. We compare the performance of distinct segmentation techniques across different image categories and shed light on 
Data collection setup



Transcribed narrative

okay looking at a face

looks like the primary lesion is a depigmented macule at the vermilion border

involving the right lower lip in the right um corner of the mouth as well as the right cutaneous lip

this is most likely vitiligo

also would consider um post-inflammatory hypopigmentation

a atypical mycosis fungoides

i am ninety percent sure that this is vitiligo

next
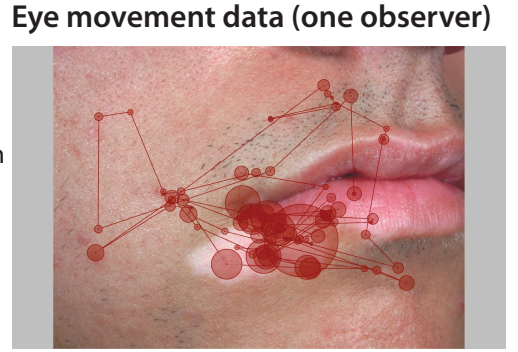

Figure 1: Left: An expert dermatologist sitting in front of the eye tracker analyzing the image and describing it aloud to the experimenter. Middle: Transcribed narrative with silent and filled pauses. Right: Eye movements overlaid on the image.

the fact that these techniques seem to better address distinct image complexity cases. This is an important finding because image application systems often use a single technique to handle heterogeneity.

3. We provide a detailed case study of various concept labels across images. This particular analysis adds deeper understanding of how a system potentially may handle a meaningful concept in an image.

4. In contrast to Vaidyanathan et al. [2015a] we report on experiment with a substantially larger dataset.

Although the proposed framework can be applied to any domain, we exemplify using dermatological images as use case of a knowledge-rich expert domain.

\section{Related Work}

In computer vision and image-based applications, automatic semantic annotation of images and image regions is highly useful but a challenging problem. Semantic understanding of images is necessary for accurate semantic annotations. Treisman and Gelade [1980] introduced semantic understanding of images in their psychological model of human visual attention. They proposed that processing of image information is a dynamic interaction between bottom-up and top-down directed processes. However, early image processing algorithms were built solely on low-level features such as color and texture to perform tasks like segmentation and retrieval [Viola and Jones 2004; Fei-Fei and Perona 2005].

In the last two decades various algorithms have been developed that successfully capture the statistics of natural scenes, identify faces, and recognize and name objects [Viola and Jones 2004; Zhang et al. 2012]. There is also an increase in sophisticated models for semantic annotation using object recognition methods [Kong et al. 2014; Zhang et al. 2012]. Although highly successful for certain applications or certain types of images these methods either failed to capture semantics with relevance to the end user's goal or fared poorly in expert domains such as medicine [Müller et al. 2004; Vinyals et al. 2014].

Our work aims at semantic image annotation by eliciting data from human experts who possess the knowledge. Several recent studies presented approaches to generate image captions and descriptions or generalize image captions [Karpathy and Fei-Fei 2014; Vinyals et al. 2014]. The method discussed in this paper differs in its integration of eye movements, spoken language descriptions, and image content. Eye movements and spoken language are non-invasive and more natural enabling us to draw out perceptual and conceptual expertise that is generally considered to be tacit knowledge [Krupinski 2000; Hoffman and Fiore 2007].
Empirical results indicate that eye movements are closely-timed with human language processing [Meyer et al. 1998; van der Meulen 2003; Griffin 2004]. This means they are not synchronous but have a small delay. Eye movements have previously been used to aid object recognition and image processing [Torralba et al. 2006]. Our work instead integrates eye movements with narrations over images and image features, thus combining perceptual, conceptual, and image information.

Roy [2000] proposed integrating vision and language elicited from infants using a mutual information model. This was useful in infant-directed interactions but is challenged when facing complex scenarios containing multiple objects/concepts such as viewing medical images. The multimodal integration problem has also been tackled for naming objects and predicting sentences using methods such as Latent Dirichlet Allocation but not on heterogeneous medical images [Li and Wang 2003; Coco and Keller 2012; Clarke et al. 2013; Yun et al. 2013]. Our approach is inspired by Yu [2004], Forsyth [2009], and Vaidyanathan et al. [2015a], who have also applied NLP methods with eye movements or image features to generate linguistic descriptions of videos, to automatically match words to the corresponding pictures, and to label medical image regions with corresponding concepts, respectively. While prior work supports the feasibility of integrating the visual and linguistic modalities, it also leaves open the question of whether established image-processing algorithms can be integrated with expert-elicited data to build an image-based application system.

\section{Data collection}

We use the dataset from Vaidyanathan et al. [2015b], which consists of audio recordings of spoken descriptions and eye movement data for 29 dermatologists, recorded while the dermatologists inspected and described 29 images of dermatological conditions. An example image is shown in Figure 1. Each image was presented to the observers on a 22 in. LCD monitor $(1680 \times 1050$ pixels) approximately $70 \mathrm{~cm}$ from the observers. A TASCAM DR-100MKII handheld recorder with a lapel microphone was used to collect the audio recordings. For the eye movement data, a Sensomotoric Instrument (SMI) RED250 eye-tracking apparatus, running at $250 \mathrm{~Hz}$, was used. Both pieces of equipment were non-intrusive in nature. Event detection used SMI BeGaze software V3.0.0X with default parameters and velocity-based (I-VT) algorithm to detect fixations. The effect of this algorithm and its parameters on the alignment is left for future work.

The data collection followed a modified Master-Apprentice procedure, which is a data elicitation methodology from the field of human-computer interaction [Beyer and Holtzblatt 1997; Li et al. 2010; Womack et al. 2012]. The experts were instructed to examine each image while moving toward a diagnosis and describe 


\section{Transcribed narrative}

okay looking at a face looks like the primary lesion is depigmented macule at the vermilion border ... sure that this is vitiligo next

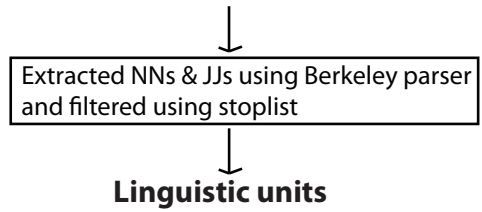

$\begin{array}{llllll}l u_{1} & l u_{2} & l u_{3} & l u_{4} & \ldots & l u_{n}\end{array}$ face primary lesion depigmented ... atypical



Figure 2: Left: Linearly ordered linguistic units obtained from the transcribed narrative. Right: Linearly ordered visual units obtained by labeling fixations using the MSFC algorithm. The labels are different when using the Grid and K-means method for identifying visual units.
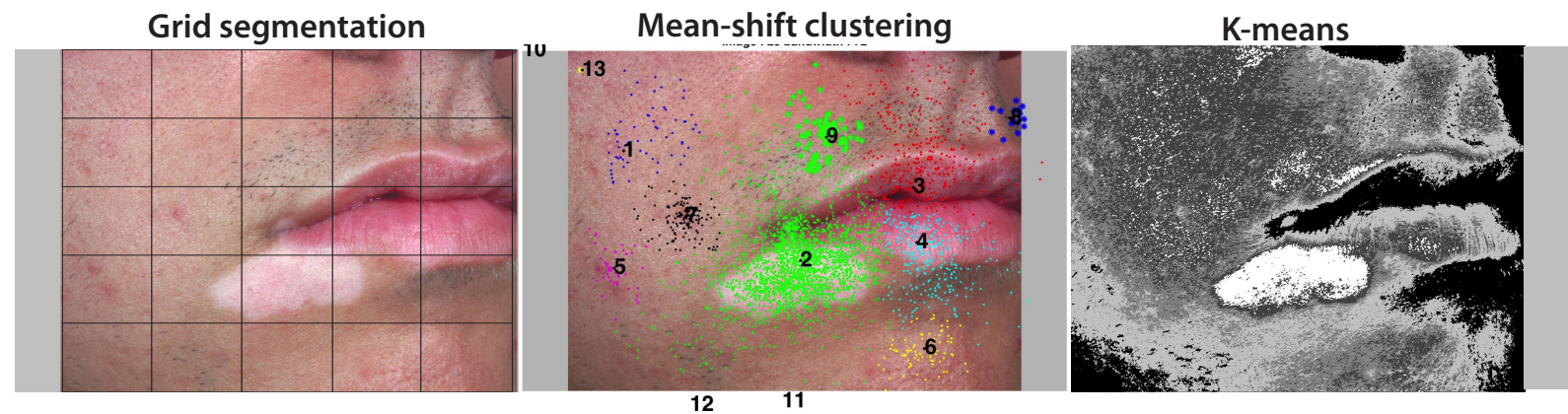

Figure 3: The three different fixation labeling techniques used to obtain visual units. Each method clusters/segments the image in a different way following which the fixations are overlaid to extract visual units

it aloud as if tutoring the experimenter. Thus, the experimenter in the data collection scenario functioned as an 'apprentice' in order to motivate the dermatologists and to help elicit rich description. Along with the descriptions, observers provided their differential diagnoses (i.e., a set of possible diagnoses), a final diagnosis, and a self-estimated certainty of their final diagnosis. Data from 3 speaker-observers were removed due to eye tracking quality reasons or other recording issues. The final used multimodal, visual-linguistic corpus consisted of 29 images and 26 experts.

\section{Alignment}

The first steps in multimodal alignment involved extracting the visual and linguistic units from the eye-tracking and spoken narratives, respectively, that would then be automatically aligned.

\subsection{Linguistic units}

The dermatologists' audio recordings were transcribed verbatim, as shown in Figure 2. Visual inspection of the transcripts revealed that the important dermatological concepts used by the experts tended to appear in noun and adjective phrases. We used the Berkeley parser to identify noun and adjective tokens [Petrov and Klein 2007].

\subsection{Visual units}

Output from the eye tracker consists of fixations given as $x, y$ coordinates and their duration as shown in Figure 2. Following Vaidyanathan et al. [2015a], we obtain visual units by encoding the fixation sequences. The encoding of the fixations is obtained using three different techniques: grid image segmentation, mean-shift fixation clustering, and K-means image segmentation. All three methods define respective segments/clusters in the image and then take advantage of the eye-tracking fixation sequence to identify the visual units that are then used in the alignment step.

The most straightforward method to segment an image is to divide it into a grid, as shown in the leftmost panel in Figure 3. Each image ( $1680 \times 1050$ pixels) is divided into a grid of 5 rows and 5 columns. Each cell in the grid is associated with a label that encodes the row and column number for that cell (e.g., $r 3 c 9, r 4 c 12$ ). The fixations of an observer are overlaid on this grid, and each fixation is labeled according to the grid cell it falls within. In this way, we obtain a linearly ordered sequence of visual units consisting of fixated image regions, encoded using the grid labels.

Visual inspection of the scanpaths of observers suggested existence of latent groups of fixations. To explore this further, we used the mean-shift fixation clustering algorithm (MSFC) [Santella and DeCarlo 2004; Vaidyanathan et al. 2015b]. It is a data-driven method that clusters fixations into "regions of interest". The advantage of using MSFC over other techniques is that MSFC does not require prior knowledge of the number of clusters and is robust to the influence of outliers. For each image, we collected fixations from all observers and performed MSFC, which assigns each fixation to a cluster, as shown in the middle panel in Figure 3. Any cluster that is outside of the image region is discarded. For each observer, we then utilize this cluster information to obtain a linearly ordered sequence of visual units (i.e., image regions determined by fixations) that acts as the other input to the alignment algorithm. An example is shown in the right panel in Figure 2. We clustered the fixations spatially, but we note that the same method 


$\begin{array}{ll}\text { face } & 2 \\ \text { face primary } & 26 \\ \text { face primary lesion } & 276 \\ \text { face primary lesion } & 276 \\ \text { face primary lesion depigmented } & 7462 \\ \text { primary lesion depigmented macule } & 4652 \\ \text { depigmented macule } & 24 \\ \text { depigmented macule vermilion } & 422 \\ \text {.. } & . \\ \text { post-inflammatory hypopigmentation } & 24 \\ \text { post-inflammatory hypopigmentaion atypical } & 242 \\ \text { hypopigmentation atypical } & 42 \\ \text { face primary lesion depigmented macule ... hypopigmentation atypical } & 327624 \ldots 2423\end{array}$

Figure 4: Example training data: A sliding window of 5 seconds is applied to the pair of visual and linguistic "sentences" to expand the data. Subsequently, contiguous visual units are merged and visual units with longest fixation duration are selected. The selected visual units, together with the linguistic units, comprise the training data.

could be used to cluster fixations temporally, a potential exploration that is left for future work.

Extending Vaidyanathan et al. [2015a], we also include the $\mathrm{K}$-means method to encode fixation sequences. We use K-means and $L a b$ color features as they have been particularly useful for dermatological images [Bosman et al. 2010; Vaidyanathan et al. 2011]. Each image is first converted into $L a b$ color space, where the $L$ channel represents illumination, the $a$ channel indicates redness-greenness, and the $b$ channel indicates blueness-yellowness in the image. Following this, a K-means algorithm is applied, resulting in a segmented image in which each pixel is labeled with the segment label it is a part of (see rightmost panel in Figure 3). Although the value of $\mathrm{K}$ can vary depending on the image and task, we chose $K=4$, since lower values miss the primary lesions present in the images. Values higher than 4 tend to over-segment many images [Vaidyanathan et al. 2011]. The fixation sequences are overlaid on the segmented image and encoded using the segment label they fall within, without loss of the linear order.

\subsection{Bitext alignment}

Studies have reported that fixations are generated before the end of words and that subjects look at an object prior to naming it [Meyer et al. 1998; van der Meulen 2003]. Additionally, it has been found that there is a temporal lag between fixations and utterances [Vaidyanathan et al. 2013]. Nevertheless, a synchronous or fixed time-delayed relationship between a fixation and the utterance with respect to that fixation is unlikely. For this reason, visual and linguistic units cannot be aligned merely by considering their temporal information. Instead, we require a method that can perform the alignment without making assumptions about the temporal relationships between the units. Conceptually, this is similar to translating one language into another, in that the structural characteristics of the source language may not parallel those of the target language. We take advantage of this insight and apply a bitext alignment approach to discover meaningful alignments of multimodal data.

In statistical machine translation (MT), word alignment models are derived using a parallel corpus of sentences in which each sentence is rendered in two different languages. In the multimodal scenario of this study, the speaker-observer's full narrative description of an image and full set of fixations on that image represent a pair of "sentences" in the training data. The small number of experts per image (26) results in a small parallel training corpus. Therefore to increase the size, we utilize a 5-second sliding window over the sentence pairs and add the linguistic and visual units within each window as a "sentence" to the training data, shown in Figure 4.
Another complication is that the sequences of visual units are substantially longer than the sequences of linguistic units. In order to balance the sequence lengths we first merge contiguous identical visual units and then select visual units that have the longest duration equal to the number of linguistic units. We select the sliding window size and fixation selection method based on the empirical study conducted by Vaidyanathan et al. [2015b]. At all times the temporal order is maintained. The obtained visual-linguistic parallel corpus serves as input for the Berkeley aligner, an EM-based word alignment package commonly used in the MT community [Liang et al. 2006]. The aligner was run with these parameter settings: 2 iterations each of IBM Model 1 and an HMM, joint training, and posterior decoding.

A gold standard reference was prepared for evaluation of the resulting multimodal alignments. An investigator with several years of experience working with medical images in the dermatology domain, as well as a reasonable understanding of both image regions and the domain vocabulary used by dermatologists, manually aligned the multimodal data for the images. Specifically, the manual alignments associated each visual unit with its corresponding linguistic units. The rest of the paper reports on results obtained when comparing against these reference alignments. Future work will involve dermatological experts' input.

\section{Results}

The example below, shows the aligner output for a given linguistic and visual "sentence" pair:

$\begin{array}{ll}\text { linguistic units } & \text { face primary lesion .. atypical } \\ \text { visual units } & 3276 . .23 \\ \text { our alignment } & \text { depigmented-2 macule-3 .. atypical-4 } \\ \text { reference alignments } & \text { depigmented-2 macule-2 .. }\end{array}$

Using the following equations we compute precision, recall, and F-measure to test how well the aligner identifies the correct word-region correspondences compared to the gold standard reference:

Precision $=\frac{A \cap S}{A}$, Recall $=\frac{A \cap S}{S}$, Fmeasure $=\frac{2 P R}{P+R}$

where $\mathrm{A}$ and $\mathrm{S}$ are number of visual-linguistic unit pairs in the output from the aligner and gold standard reference data, respectively. The average alignment results over all of the images are compared with two temporal baselines in Table 1. One baseline assumes that an observer utters the word corresponding to a region at the moment his/her eyes fixate on that region. The second baseline assumes that there is a 1-second [Griffin 2013] delay between a fixation and the utterance of the word corresponding to 


\begin{tabular}{|l|c|c|c|c|c|c|c|c|c|}
\hline & \multicolumn{3}{|c|}{ Grid-based } & \multicolumn{3}{c|}{ MSFC } & \multicolumn{3}{c|}{ K-means } \\
\hline & Baseline & $\begin{array}{c}\text { 1-sec delay } \\
\text { Baseline }\end{array}$ & Ours & Baseline & $\begin{array}{c}\text { 1-sec delay } \\
\text { Baseline }\end{array}$ & Ours & Baseline & $\begin{array}{c}\text { 1-sec delay } \\
\text { Baseline }\end{array}$ & Ours \\
\hline Precision & 0.32 & 0.34 & 0.38 & 0.36 & 0.38 & $\mathbf{0 . 4 5}$ & 0.39 & 0.4 & 0.41 \\
\hline Recall & 0.27 & 0.28 & 0.29 & 0.44 & 0.44 & $\mathbf{0 . 5 6}$ & 0.44 & 0.44 & $\mathbf{0 . 5 6}$ \\
\hline F-measure & 0.28 & 0.3 & 0.32 & 0.39 & 0.39 & $\mathbf{0 . 4 9}$ & 0.41 & 0.41 & 0.46 \\
\hline
\end{tabular}

Table 1: Comparison of alignment performance, average across images. The proposed aligner, specially using MSFC performs the best.

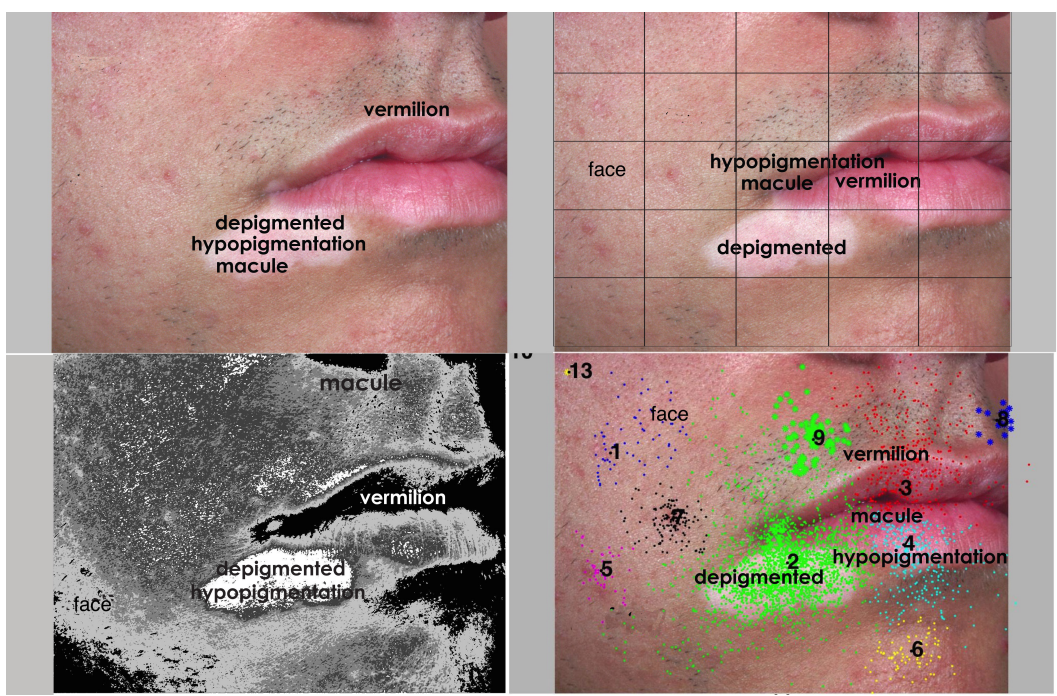

Figure 5: Annotations from Top-Left: The investigator, Top-Right: Grid-based, Bottom-Left: K-means, and Bottom-Right: MSFC alignments, respectively. Compared to the investigator's reference alignments, 'hypopigmentation' and 'vermilion' although incorrectly placed, are still close to their corresponding regions with MSFC. In contrast 'macule' with K-means is quite far from where it should be.

that region. Since the set of regions and their labels change with the segmentation technique being used each segmentation method has its own set of exact and delayed baselines, reference alignment, and proposed alignment. The delayed baseline tends to perform as well as or better than the exact match baseline.

Our alignment method, irrespective of the fixation encoding technique, yields stronger performance in comparison to the two baselines relying on exact and fixed-delay time match between words and fixations. In general, within our alignment method both MSFC and K-means outperform the grid method. MSFC achieves absolute improvement of $7 \%, 12 \%$, and $10 \%$ for precision, recall and F-measure, respectively, over the delayed baseline. K-means on the other hand when compared to the delayed baseline achieves $1 \%, 12 \%$, and $5 \%$ absolute improvement for precision, recall, and F-measure, respectively. The results hold on a per-image basis as well, with the MSFC alignment approach yielding higher recall and F-measure than baselines in 29 and 28 of the total 29 images, respectively, and higher precision than baselines in 24 of the 29 images. The K-means linked alignment on the other hand yields comparable precision for only 17 of the 29 images.

Figure 5 shows an image overlaid with the most frequently used linguistic units to which those regions were aligned by our aligner using the three methods. We compare it to the image when labeled by the investigator in Figure 5 Top-Left. The labels are generally accurate and well located on the image in the K-means and MSFC cases when compared to the manually annotated image. The grid-based method performs poorly with only one correct label-region association. Labels such as hypopigmentation, although incorrect, are still close to the corresponding region in the case of MSFC for this image.

We organized our images into four different groups following Li et al. [2013]: single lesion, multiple lesions, bilateral lesions, and distributed lesions (Figure 6). The category multiple lesions had almost double the number of images in comparison to the other three categories. Few images in our database posed ambiguity regarding which category they belonged to. Interestingly, our results indicate that for most of the single, bilateral, and distributed lesions cases MSFC with bitext alignment performs better than K-means whereas annotations for images with multiple lesions scattered around are mostly improved when using K-means with bitext alignment. Figure 6 shows some annotations achieved for different cases using MSFC (black) and K-means (red).

Further investigation reveals that even though MSFC performance for multiple lesion cases is lower than that of K-means, the best improvement ( $15 \%$ in $\mathrm{F}$-measure) that our aligner achieves over the delayed baseline is for these images with multiple lesions. As already noted in Vaidyanathan et al. [2015b]. The best improvement ( $10 \%$ in F-measure) over the delayed baseline for the $\mathrm{K}$-means method is for images with single lesions.

We also analyzed the performance of the aligner for certain concept labels across images. In dermatology the primary lesion present in an image is called the primary morphology. Across the 29 images, 9 primary morphologies were represented: plaque, papule, nodule, patch, pustule, bulla, macule, vesicle and hypopigmentation with plaques being most frequent and hypopigmentation being the least frequent. Each of these primary morphologies was either present independently or in groups with others. For each label, we 


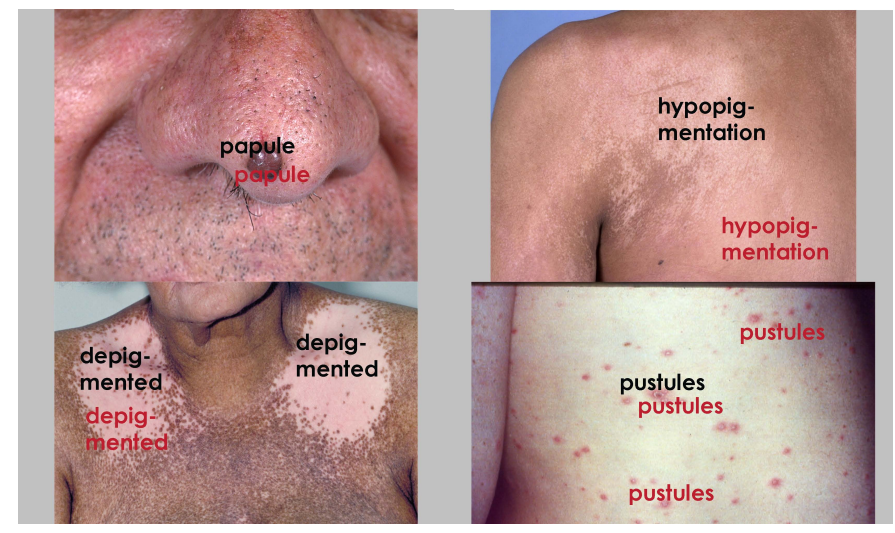

Figure 6: Annotation examples for four different kinds of images with MSFC (black) and K-means (red) used to obtain visual units. Top-Left: Single lesion case where both MSFC and K-means are comparable with the reference alignment, Top-Right: Distributed lesion case where MSFC correctly identifies 'hypopigmentation', Bottom-Left: Bilateral lesions where MSFC correctly annotates both parts of the lesion whereas K-means fails to annotate one part, and Bottom-Right: Multiple lesions case where K-means correctly labels all the regions pertaining to 'pustules' and MSFC labels only one. This shows the advantage and disadvantage of using each method.

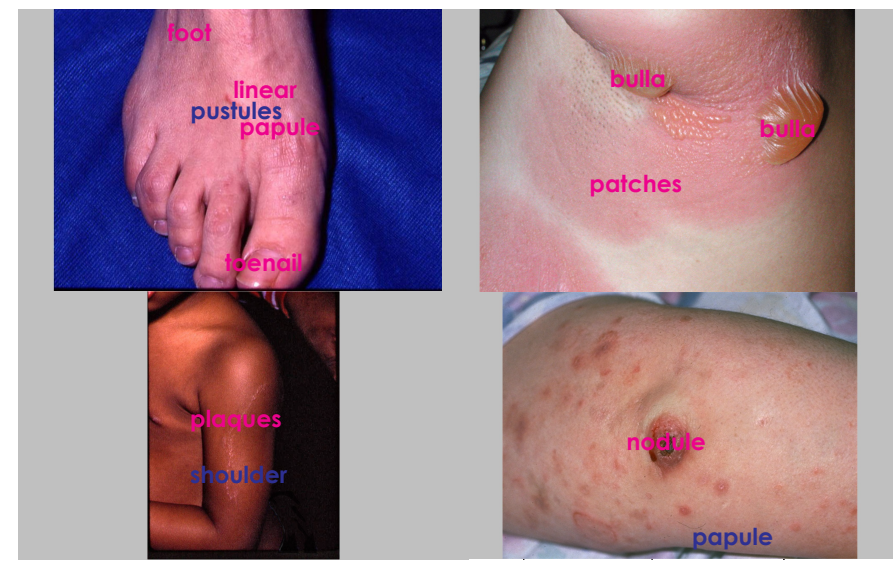

Figure 7: Correct and incorrect labels identified by MSFC. Many labels are correctly (pink) aligned even in challenging cases. The incorrect labels are highlighted in blue. Top-Left: Whereas tiny 'pustules' (on second toe) are incorrectly identified 'linear' and 'papule' are satisfactory. Top-right: The system correctly identifies the labels 'patches' and 'bulla' but does not recognize the tiny collection of shiny 'vesicles' underneath the arm. Bottom-Left: Body part 'shoulder' although incorrectly annotated it is still identified as a part of the arm. Bottom-right: While the single lesion 'nodule' is correctly identified the many scattered 'papule' are incorrectly annotated.

calculated precision for each of these labels across all of the images for the MSFC and K-means method. Precision for MSFC in general tended to be higher than that for K-means, particularly for papule and nodule (0.9). Precision for the rest of the labels ranged from 0.65 to 0.82 for MSFC. An in-depth analysis showed that even though K-means correctly aligned many of these 9 labels, it also aligned them with many non-relevant regions thereby lowering the precision. Using the K-means method, pustule and vesicle scored slightly better $(0.75)$ than using MSFC.

\section{Discussion}

The alignment results, for all three visual unit identification techniques, improved on the baselines, supporting that it would be naive to assume one-to-one or fixed-delay correspondence between utterances and eye movements, and underscoring the promise of our alignment-annotation approach in this study. Considering highest scores on metrics the MSFC method outperforms the grid method in all cases and K-means in many cases. While both MSFC and $\mathrm{K}$-means use fixations as pointers when identifying sequences of visual units from the determined image regions, the major difference is that MSFC identifies the regions by clustering users' fixations based on space without using image features whereas $\mathrm{K}$-means identifies the image regions based on image features without using fixations. Even though K-means in comparison to MSFC uses image features in addition to fixations, it does not use the fixations during the segmentation process. The performance when considering MSFC suggests that we need to include perceptual information to capture the semantics of images from users' perspectives for the process of identifying image regions. Likewise the lower performance of MSFC on images with scattered multiple lesions indicates that an image-based framework is incomplete without including image features in segmenting out regions then considered as candidate visual units.

Both MSFC and K-means in Figure 6 do well for the single lesion image on top-left but in the multiple lesion image, MSFC does worse than K-means (bottom-right). In this case, MSFC is able to annotate one particular region as pustule but K-means, on the other hand, is able to identify all regions associated with pustule. When the lesion size gets larger, the K-means method either fails to annotate all of the regions, as in the bilateral case (bottom-left) 
or annotates the incorrect ones, as in the distributed lesion case (top-right). A visual inspection of the $\mathrm{K}$-means segmented images indicates that, in some cases, color features alone are insufficient and that texture might be playing an important role as well, as shown in Figure 6 Top-Right where color is not as strong a feature as texture for differentiating the lesion. To remedy this, in the future our framework will include both color and texture features in conjunction with eye movements to obtain better annotations. Results suggest that a framework should include distinct techniques to addresses the heterogeneity of regions across images.

Figure 7 shows more annotation examples from our database using the MSFC method. It correctly identifies single lesions bulla, nodule, patches in the Top-Right and Bottom-right panels but misses multiple small papules in the Bottom-Right. The Top-Left example is an interesting case where MSFC identifies the label papule, probably because the papules form one long lesion, as opposed to Bottom-right where they are scattered. This is confirmed with the observation that MSFC fails to identify pustules in Top-Left since they are scattered (on the second toe).

The lower performance of MSFC on images with multiple lesions could be due to that fixations are single image coordinates and sometimes can lie just outside of the tiny multiple lesions, even though the observer might have been paying attention to the lesion itself. This could be solved by considering a region around the fixation approximating the fovea. Also, some of the images with distributed lesions have values very close to the skin in the Lab space. This may lead to a poor K-means segmentation output thereby resulting in lower performance of the aligner when compared to the MSFC method. We will address this issue by exploring more sophisticated hierarchical segmentation algorithms.

For both MSFC and K-means there is substantial improvement in the recall values over baselines when using bitext alignment across all evaluated image cases. We note that precision is often lower due to nouns and adjectives (atypical) that are not physically present in the image. Additionally there are some linguistic units that correspond to the entire image rather than a specific region (e.g., face as shown in Figure 5). Such holistic or abstract units are not included in the manually derived reference alignments, resulting in lower alignment precision for these images. We also note that although the researcher who produced the reference alignments for this paper has knowledge of dermatology, the alignments that would be generated by a dermatology expert could potentially be different.

Therefore, it can be summarized that key labels such as papule and nodule had high precision for MSFC whereas pustule and vesicle had high-precision for K-means. This indirectly confirms the observation that MSFC performed well on single lesion images since most of the single lesion images had large papule or nodule as opposed to groups of tiny pustules or vesicles, in which case $\mathrm{K}$-means tends to perform better. The in-depth analysis of primary morphology labels across images is useful in identifying their characteristics across images. We could potentially extract all the regions from all the images pertaining to each of these labels, extract image features and learn both common and idiosyncratic features for each label.

\section{Conclusions and future work}

Our results continue to support that 1-to-1 or fixed-delay temporal correspondence between the two modalities is unlikely. In addition, the individual performances of MSFC and K-means show that both methods have strengths that together could be of more value than when individually used. One way to combine the two techniques is to identify the cluster (multiple, single, bilateral, distributed) of an image and select a method (MSFC, K-means, etc.) that performs well on the identified cluster type. It may also be useful to weigh the output of certain methods for particular concept labels.

Our existing framework could further be improved by incorporating knowledge about conceptual relations such as meronymy (part-whole relations) in both linguistic and visual modalities. For example, in Figure 6, the word nasal corresponds to broader image regions, whereas papule corresponds to an internal region. In this particular case, there is a nasal has-a papule relation. We intend to use hierarchical image segmentation and a medical ontology to obtain part-whole and other lexical semantic relationships. As $\mathrm{K}$-means is a pixel-based clustering technique we will investigate other advanced segmentation techniques such as region-based or graph-cut segmentation methods.

In future work, the obtained annotations will be used to map low-level image features from these images and image regions to the aligned domain concepts. A model using these mappings could be applied towards semantic annotations for unseen images for applications such as image retrieval and classification.

\section{Acknowledgements}

This work was supported by NIH and NSF. We also thank the reviewers, Dr. Calvelli, and Logical Images Inc. Parts of Figure 1 appeared in Vaidynathan et al. [2015b].

\section{References}

Beyer, H., And Holtzblatt, K. 1997. Contextual design: Defining customer-centered systems. Elsevier, San Diego.

Bosman, H., PetKov, N., And Jonkman, M. 2010. Comparison of color representations for content-based image retrieval in dermatology. Skin Research and Technology 16, 1, 109-113.

Clarke, A. D., Coco, M. I., And Keller, F. 2013. The impact of attentional, linguistic, and visual features during object naming. Frontiers in Psychology 4.

Coco, M. I., AND Keller, F. 2012. Scan patterns predict sentence production in the cross-modal processing of visual scenes. Cognitive Science 36, 7, 1204-1223.

FeI-Fei, L., And Perona, P. 2005. A bayesian hierarchical model for learning natural scene categories. In IEEE Conference on Computer Vision and Pattern Recognition, vol. 2, IEEE, 524-531.

Forsyth, D. A., Berg, T., Alm, C. O., Farhadi, A., Hockenmaier, J., LoefF, N., AND WANG, G. 2009. Words and pictures: Categories, modifiers, depiction, and iconography. In Object Categorization: Computer and Human Vision Perspectives, S. Dickinson, M. Tarr, A. Leonardis, and B. Shiele, Eds. Cambridge University Press, Cambridge, $167-181$.

Goldstone, R. 1998. Perceptual learning. Annual Review of Psychology 49, 1, 585-612.

GRIFFIN, Z. M. 2004. Why look? Reasons for eye movements related to language production. The interface of language, vision, and action: Eye movements and the visual world, 213-247.

GRIFFIN, Z. M. 2013. Compared to generating an utterance, comprehending one is a piece of. The interface of language, vision, and action: Eye movements and the visual world, 213. 
Hoffman, R., And Fiore, S. 2007. Perceptual (re) learning: A leverage point for human-centered computing. Intelligent Systems, IEEE 22, 3, 79-83.

Karpathy, A., And FEI-FeI, L. 2014. Deep visual-semantic alignments for generating image descriptions. arXiv preprint arXiv:1412.2306.

Kong, C., Lin, D., Bansal, M., URTasun, R., AND Fidler, S. 2014. What are you talking about? Text-to-image coreference. In IEEE Conference on Computer Vision and Pattern Recognition, 3558-3565.

KRUPINSKI, E. 2000. The importance of perception research in medical imaging. Radiation Medicine 18, 6, 329-334.

Kuznetsova, P., Ordonez, V., Berg, A. C., Berg, T. L., AND CHOI, Y. 2013. Generalizing image captions for image-text parallel corpus. In Proceedings of ACL, 790-796.

LI, J., AND WANG, J. Z. 2003. Automatic linguistic indexing of pictures by a statistical modeling approach. IEEE Transactions on Pattern Analysis and Machine Intelligence 25, 9, 1075-1088.

Li, R., Vaidyanathan, P., Mulpuru, S., Pelz, J. B., Shi, P., Calvelli, C., AND HaAke, A. R. 2010. Human-centric approaches to image understanding and retrieval. In Western New York Image Processing Workshop (WNYIPW), 62-65.

LI, R., ShI, P., AND HAAKE, A. R. 2013. Image understanding from experts' eyes by modeling perceptual skill of diagnostic reasoning processes. In IEEE Conference on Computer Vision and Pattern Recognition, 2187-2194.

Liang, P., Taskar, B., And Klein, D. 2006. Alignment by agreement. In Proceedings of NAACL-HLT, 104-111.

LiU, Y., Zhang, D., LU, G., AND MA, W.-Y. 2007. A survey of content-based image retrieval with high-level semantics. Pattern Recognition 40, 1, 262-282.

Meyer, A. S., Sleiderink, A. M., Levelt, W. J., et Al. 1998. Viewing and naming objects: Eye movements during noun phrase production. Cognition 66, 2, B25-B33.

MÜLler, H., MichouX, N., BANdon, D., ANd Geissbuhler, A. 2004. A review of content-based image retrieval systems in medical applications-clinical benefits and future directions. International Journal of Medical Informatics 73, 1, 1-23.

Petrov, S., AND Klein, D. 2007. Improved inference for unlexicalized parsing. In Proceedings of HLT-NAACL, 404-411.

RoY, D. 2000. Integration of speech and vision using mutual information. In Proceedings of ICASSP, 2369-2372.

Santella, A., And DeCarlo, D. 2004. Robust clustering of eye movement recordings for quantification of visual interest. In Proceedings of the 2004 Symposium on Eye Tracking Research \& Applications, 27-34.

Shanteau, J. 1992. How much information does an expert use? Is it relevant? Acta Psychologica 81, 1, 75-86.

Smeulders, A. W. M., Worring, M., Santini, S., Gupta, A., AND JAIN, R. 2000. Content-based image retrieval at the end of the early years. IEEE Transactions on Pattern Analysis and Machine Intelligence 22, 12, 1349-1380.

Socher, R., Karpathy, A., Le, Q. V., Manning, C. D., AND NG, A. Y. 2014. Grounded compositional semantics for finding and describing images with sentences. Transactions of the Association for Computational Linguistics 2, 207-218.
Thomason, J., Venugopalan, S., Guadarrama, S., SAENKO, K., AND MOONEY, R. 2014. Integrating language and vision to generate natural language descriptions of videos in the wild. In Proceedings of COLING 2014, the 25th International Conference on Computational Linguistics: Technical Papers, Dublin City University and Association for Computational Linguistics, Dublin, Ireland, 1218-1227.

Torralba, A., Oliva, A., Castelhano, M. S., AND Henderson, J. M. 2006. Contextual guidance of eye movements and attention in real-world scenes: The role of global features in object search. Psychological Review 113, 4, 766.

Treisman, A. M., AND Gelade, G. 1980. A feature-integration theory of attention. Cognitive Psychology 12, 1, 97-136.

Vaidyanathan, P., Pelz, J. B., Li, R., Mulpuru, S., Wang, D., Shi, P., Calvelli, C., AND HaAke, A. R. 2011 Using human experts' gaze data to evaluate image processing algorithms. In IVMSP Workshop, IEEE, 129-134.

Vaidyanathan, P., Pelz, J. B., Alm, C. O., Calvelli, C., ShI, P., AND HaAKe, A. R. 2013. Integration of eye movements and spoken description for medical image understanding. In Proceedings of the 17th European Conference on Eye Movements, 45-46.

Vaidyanathan, P., Prudhommeaux, E., Alm, C. O., Pelz, J. B., AND HAAKE, A. R. 2015a. Alignment of eye movements and spoken language for semantic image understanding. In IWCS 2015, ACL, 76-82.

Vaidyanathan, P., Prudhommeaux, E., Alm, C. O., And PELZ, J. B. 2015b. Computational integration of human vision and natural language through bitext alignment. In $(i V \& L 2015$ at, Empirical Methods on Natural Language Processing, ACL, $76-82$.

VAN DER Meulen, F. F. 2003. Coordination of eye gaze and speech in sentence production. Trends in Linguistics Studies and Monographs 152, 39-64.

Vinyals, O., Toshev, A., Bengio, S., And Erhan, D. 2014. Show and tell: A neural image caption generator. arXiv preprint arXiv:1411.4555.

Viola, P., AND Jones, M. J. 2004. Robust real-time face detection. International Journal of Computer Vision 57, 2, $137-154$.

Womack, K., McCoy, W., Alm, C. O., Calvelli, C., Pelz, J. B., Shi, P., And HaAKe, A. R. 2012. Disfluencies as extra-propositional indicators of cognitive processing. In Proceedings of the Workshop on Extra-Propositional Aspects of Meaning in Computational Linguistics, ACL, 1-9.

Yu, C., AND BAllard, D. H. 2004. A multimodal learning interface for grounding spoken language in sensory perceptions. ACM Transactions on Applied Perception (TAP) 1, 1, 57-80.

Yun, K., Peng, Y., Adeli, H., Berg, T., Samaras, D., And ZELINSKY, G. 2013. Specifying the relationships between objects, gaze, and descriptions for scene understanding. Journal of Vision 13, 9, 1309-1309.

Zhang, D., Islam, M. M., AND LU, G. 2012. A review on automatic image annotation techniques. Pattern Recognition 45, $1,346-362$. 